\title{
Molecular Imaging of PARP
}

\author{
Brandon Carney*1,2, Susanne Kossatz*1, and Thomas Reiner ${ }^{1,3}$ \\ ${ }^{1}$ Department of Radiology, Memorial Sloan Kettering Cancer Center, New York, New York; ${ }^{2}$ Department of Chemistry, \\ Hunter College, and PhD Program in Chemistry, Graduate Center of City University of New York, New York, New York; \\ and ${ }^{3}$ Department of Radiology, Weill Cornell Medical College, New York, New York
}

The poly(adenosine diphosphate-ribose)polymerase (PARP) family of enzymes is an important factor in the cellular DNA damage response and has gained much attention for its role in many diseases, particularly cancer. Targeted molecular imaging of PARP using fluorescent or radiolabeled tags has followed on the success of therapeutic inhibitors and gained momentum over the past few years. This review covers PARP imaging from the very first imaging agents up to the current state of the technology, with a focus on the clinical applications made possible by these agents.

Key Words: optical; PET; SPECT; PARP; fluorescence; translation

J Nucl Med 2017; 58:1025-1030

DOI: 10.2967/jnumed.117.189936

The poly(adenosine diphosphate-ribose)polymerase (PARP) enzyme family — more specifically, PARP1-is an important participant in the DNA damage response. It is upregulated in many malignancies and has been identified as a critical component for cellular survival. PARP inhibitors have shown therapeutic potential, either in conjunction with DNAdamaging agents or as monotherapies in tumors with defects in other DNA repair pathways (1-3). The success of PARP inhibitor therapy led to Food and Drug Administration approval of olaparib in 2014, rucaparib in 2016, and niraparib in 2017. Other PARP inhibitors are in late-stage clinical trials for a variety of malignancies.

The development of PARP imaging agents opens up a new realm of possibilities in the clinic. Optical imaging agents might be used for both diagnostic purposes and improvement of surgical procedures through intraoperative imaging. Radiolabeled probes allow direct measurement of PARP expression in patients, with the goal of improving stratification of patients, quantification of target engagement by therapeutic inhibitors, and monitoring of noninvasive treatment.

Received Mar. 8, 2017; revision accepted Apr. 26, 2017.

For correspondence or reprints contact: Thomas Reiner, Department of Radiology, Memorial Sloan Kettering Cancer Center, 1275 York Ave., New York, NY 10065.

E-mail: reinert@mskcc.org

${ }^{*}$ Contributed equally to this work.

Published online May 4, 2017.

COPYRIGHT (C) 2017 by the Society of Nuclear Medicine and Molecular Imaging.
This review focuses on radiolabeled and fluorescent PARP imaging agents. More details about PARP radiotracers from the larger perspective of imaging the DNA damage response can be found in an excellent review by Knight et al. (4). More details about the underlying biology can be found in reviews by Scott et al. (1), Michels et al. (2), and Pommier et al. (3). Here, we seek to present the current state of the art of optical and radioactive PARP imaging agents and their ultimate clinical goals.

\section{BODIPY-FL-LABELED PARP IMAGING AGENTS}

The development of optical PARP agents is challenged by the intranuclear localization of PARP1 and PARP2. Hence, imaging requires targeting vectors that can freely cross both the cell membrane and the nuclear envelope and do not lose this ability after their conjugation with a fluorescent dye.

The most published optical PARP reporter, the fluorescent PARP1 inhibitor (PARPi-FL) (5-16) (half-maximal inhibitory concentration $\left.\left[\mathrm{IC}_{50}\right], 12.2 \mathrm{nM}\right)$, is structurally similar to the Food and Drug Administration-approved olaparib (17) $\left(\mathrm{IC}_{50}\right.$, $5.0 \mathrm{nM}(18)$ ). The fluorescence signal of PARPi-FL stems from the dye BODIPY-FL (excitation maximum/emission maximum, 503/512 nm), which was covalently linked to the olaparib scaffold (Fig. 1) (13). High-contrast imaging of PARP1-expressing nuclei in vitro and in vivo implies high cell permeability and rapid washout of unbound PARPi-FL $(7,9,11,13,16)$, indicating that the dye conjugation and resulting increase in molecular weight (PARPi-FL, $640 \mathrm{~g} / \mathrm{mol}$; olaparib, $435 \mathrm{~g} / \mathrm{mol}$ ) do not strongly perturb the molecule's binding affinity and cell permeability. Uptake specificity was shown by correlation of PARP1 protein expression and PARPi-FL retention, as well as by the ability to block PARPiFL uptake by saturating PARP with olaparib (13). One set of studies $(7,8)$ presented PARPi-FL as a tool to measure drugtarget interaction in real time at subcellular resolution in vitro and in vivo using multiphoton fluorescence anisotropy microscopy. The anisotropy value of an excited fluorophore is linked to its mobility and hence provides insight into its binding state. The earlier study (7) showed anisotropy measurements of PARPi-FL in vivo using a window chamber model. Later (8), an indirect anisotropy approach was used to measure pharmacodynamic parameters of olaparib, allowing the identification of single cells that featured a low occupancy despite a high average target engagement. 


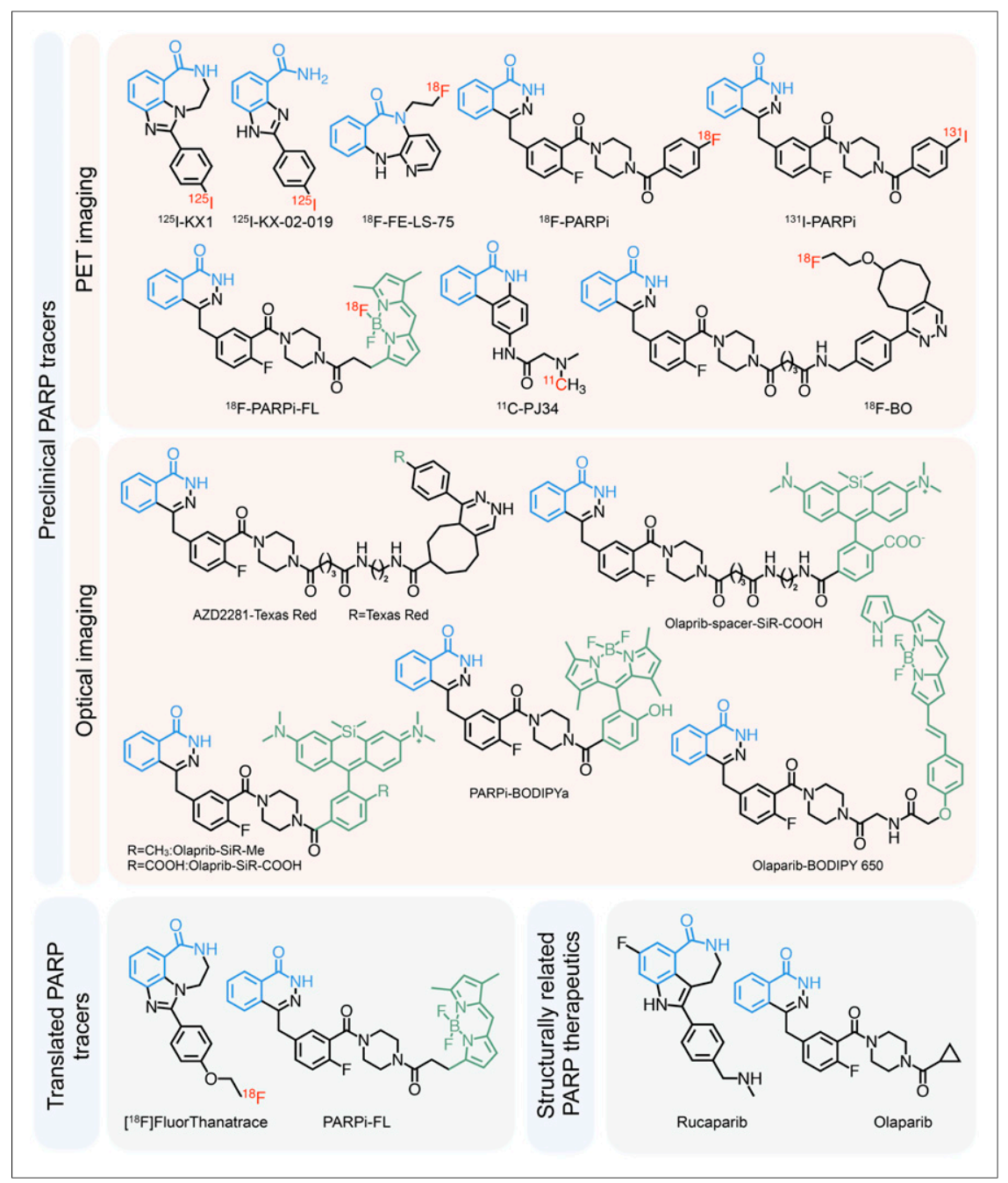

FIGURE 1. PARP inhibitors for imaging. Nicotinamide-mimicking benzamide moiety is highlighted in blue, fluorescent moieties in green, and radioactive nuclides in red.

Another study (5) presented an activatable version of PARPi-FL in which an inactive, photocaged BODIPY dye was conjugated to the olaparib scaffold (PARPi-BODIPYc; $\left.\mathrm{IC}_{50}, 32 \mathrm{nM}\right)$. Ultraviolet light ( $350-410 \mathrm{~nm}$ ) converted it to its fluorescent version, PARPi-BODIPYa $\left(\mathrm{IC}_{50}, 68 \mathrm{nM}\right)$ by photolytic cleavage of the 2,6-dinitrobenzyl caging group. Up to $3 \mathrm{~h}$ of washing were required to receive a crisp nuclear signal of PARPi-BODIPYa, compared with 10 min for PARPi-FL, indicating decreased cell permeability and clearance (Fig. 2A).

PARPi-FL is the only optical PARP imaging agent that has been verified to accumulate in tumors in vivo. The first study (9) to do so used PARPi-FL to image U87 and U251 orthotopic and subcutaneous glioblastoma xenografts ex vivo and found tumor-to-brain and tumor-to-muscle ratios of more than 10 at $1 \mathrm{~h}$ after intravenous injection, suggesting that PARPi-FL could be useful for intraoperative imaging of glioblastoma. PARPi-FL was also used for imaging of oral squamous cell carcinoma. One study (10) found increased PARPi-FL uptake $48 \mathrm{~h}$ after irradiation, which correlated with an increased PARP1 expression, suggesting that tumor response to radiation can be quantified. Another study (11) showed specific high-contrast imaging (tumor-to-muscle/trachea/tongue ratios $>3$ ) in orthotopic oral squamous cell carcinoma models 90 min after injection using clinical imaging instruments including a fluorescence endomicroscope and a surgical stereoscope (Fig. 2B). This study also showed the feasibility of topical application of PARPi-FL, which accumulated in oral squamous cell carcinoma tumors after a 2-min application interval, and reported penetration and nuclear accumulation of PARPi-FL to a depth of more than $250 \mu \mathrm{m}$ in tumor tissue (Fig. 2C).

\section{OPTICAL IMAGING OF PARP IN THE NEAR-INFRARED SPECTRUM}

PARPi-FL is limited by low tissue penetration of the BODIPY-FL fluorescence $(502 / 510 \mathrm{~nm})$. Imaging in the near-infrared spectrum $(600-900 \mathrm{~nm})$ would allow for improved tissue penetration and therefore a higher signalto-background ratio. AZD2281-Texas Red (Life Technologies) (19) ( $\mathrm{IC}_{50}$, $15.4 \mathrm{nM} ; 596 / 615 \mathrm{~nm}$ ) was produced by bioorthogonal trans-cyclooctene (TCO)/tetrazine cycloaddition. In vitro imaging showed nuclear accumulation after addition of first Texas Red-tetrazine and then AZD2281-trans-cyclooctene (Fig. 2A), whereas a prereacted fullsized AZD2281-Texas Red (1,536 $\mathrm{g} / \mathrm{mol}$ ) showed reduced cell permeation. A study (20) that adapted this modular approach using AZD2281-trans-cyclooctene and Cy5-tetrazine also showed in vitro nuclear staining but did not report $\mathrm{IC}_{50}$ values. A labeling strategy similar to that for PARPi-FL produced olaparib-BODIPY650 (16) (IC $50,92 \mathrm{nM}$; 646/660 nm; $895 \mathrm{~g} / \mathrm{mol}$ ). The increase in size and hydrophilicity (cLogP, 6.0; PARPi-FL cLogP, 4.0) presumably affected permeability and off-target clearance, requiring in vitro washing intervals of $2 \mathrm{~h}$ to visualize the nuclear staining (Fig. 2A).

Charge was also reported as a significant factor in cellular distribution (21). One study labeled olaparib with a new class of cell-permeable silicon-containing rhodamines ( $\mathrm{SiR}, 653 / 670 \mathrm{~nm}$ ). These contained either a methyl group or a carboxyl group conjugated directly (SiR-COOH) or via a short aliphatic spacer (SiR-spacer-COOH). OlaparibSiR-methyl ( $\mathrm{IC}_{50}, 99 \mathrm{nM}$; molecular weight, $792 \mathrm{~g} / \mathrm{mol}$; cLogP, 4.23) was cell-permeable but accumulated only in mitochondria. The negatively charged olaparib-SiR-COOH $\left(\mathrm{IC}_{50}, 100 \mathrm{nM}\right.$; molecular weight, $822 \mathrm{~g} / \mathrm{mol}$; cLogP, 3.74) showed cytoplasmic staining comparable to that of 


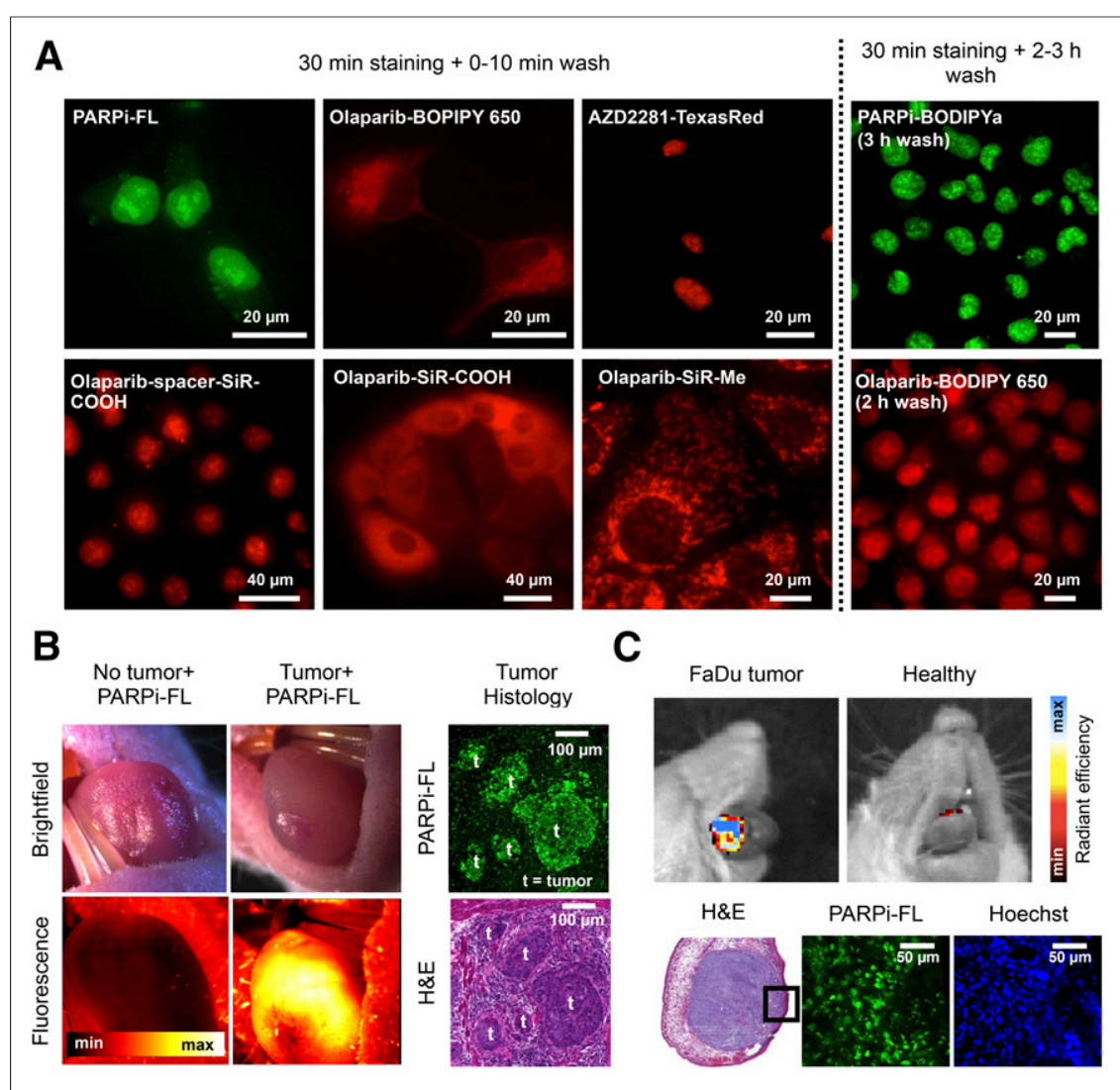

FIGURE 2. Fluorescent PARP imaging agents $(5,11,16,21)$. (A) In vitro staining with various fluorescent PARP inhibitors. (B) In vivo imaging of orthotopic oral squamous cell carcinoma with PARPi-FL using fluorescence stereoscope, confirmed by histology. (C) In vivo imaging of orthotopic oral squamous cell carcinoma xenografts after a 2-min topical application of PARPi-FL. H\&E = hematoxylin and eosin.

olaparib-BODIPY 650. However, olaparib-spacer-SiR-COOH $\left(\mathrm{IC}_{50}, 112 \mathrm{nM}\right.$; molecular weight, $978 \mathrm{~g} / \mathrm{mol}$; cLogP, 3.59) allowed imaging of nuclear PARP1, following a standard PARPi-FL staining protocol with a short wash (Fig. 2A). This indicates that neutralization of the negative charge of $\mathrm{SiR}-\mathrm{COOH}$ and increased hydrophobicity introduced by the spacer outweighed the handicap of the higher molecular weight.

In conclusion, retention of fast cell permeation and rapid clearance of optical PARP inhibitors is most essential for their performance. Although in vitro PARP1 staining has been shown for the near-infrared probes AZD2281-Texas Red and olaparib-spacer-SiR-COOH, their in vivo performance remains unknown. Despite the general trend to move toward the near-infrared spectrum for optical imaging, in the specific case of PARP1 imaging, BODIPY-FL labeling currently remains the most promising approach. Its brightness, resulting from a high maximum absorption and high quantum yield, could prove advantageous even over most near-infrared fluorochromes when considering intraoperative imaging and detection of surface tumors for which limited tissue penetration does not impede the diagnostic value.

\section{THE FIRST RADIOLABELED PROBES}

The first radiolabeled PARP inhibitor was developed for imaging the PARP1 hyperactivation in type I diabetesrelated necrosis. ${ }^{11} \mathrm{C}-\mathrm{PJ} 34$ (22) was based on PJ34 ( $\left.\mathrm{IC}_{50}, 170.2 \mathrm{nM}(23)\right)$, which showed promise as a therapeutic agent for diabetes-related endothelial dysfunction (24). A rat model of type I diabetes showed higher uptake of ${ }^{11} \mathrm{C}-\mathrm{PJ} 34$ in the liver and pancreas of treated animals than in untreated controls, indicating that ${ }^{11} \mathrm{C}-\mathrm{PJ} 34$ is capable of detecting disease-related increased PARP1 activity.

The first ${ }^{18} \mathrm{~F}$-labeled PARP inhibitor, ${ }^{18} \mathrm{~F}-\mathrm{FE}$ LS 75 (inhibitor constant $\left.\left[\mathrm{K}_{\mathrm{i}}\right], 200 \mathrm{nM}\right)(25)$, was designed to investigate the potential of LS-75 $\left(\mathrm{K}_{\mathrm{i}}\right.$, $18 \mathrm{nM}$ ) to act as a neuroprotective agent by regulating PARP1 activity. ${ }^{18} \mathrm{~F}-\mathrm{FE}$ LS 75 was stable in plasma over 120 min, but no further biologic studies have been published.

\section{RADIOLABELED PROBES STRUCTURALLY SIMILAR TO OLAPARIB}

The first radiolabeled PET imaging PARP inhibitor with structural similarity to olaparib was ${ }^{18} \mathrm{~F}-\mathrm{BO}\left(\mathrm{IC}_{50}\right.$, $17.9 \mathrm{nM}$ ). Analogous to the fluorescent compounds, the cyclopropyl group of olaparib was replaced with an ${ }^{18} \mathrm{~F}-$ labeled moiety. The probe was synthesized by trans-cyclooctene/tetrazine bioorthogonal click chemistry and purified either by conventional semipreparative high-performance liquid chromatography (26) or with the aid of magnetic trans-cyclooctene functionalized scavenging beads (27). In vitro experiments in breast cancer cells showed higher uptake of ${ }^{18} \mathrm{~F}-\mathrm{BO}$ in MDA-MB-436 cells than in MDAMB-231 cells, and the uptake coincided with PARP1 expression. MDA-MB-436 xenografts showed an SUV of 0.9, and specificity was shown by blocking with a $100 \mathrm{mg} / \mathrm{kg}$ dose of olaparib $12 \mathrm{~h}$ before ${ }^{18} \mathrm{~F}-\mathrm{BO}$ imaging, which reduced the SUV to $0.4 .{ }^{18} \mathrm{~F}-\mathrm{BO}$ uptake also correlated with PARP1 expression in ovarian (SKOV3, A2780) and pancreatic (MIAPaCa-2, PANC-1) tumor models (13). To evaluate the potential of ${ }^{18} \mathrm{~F}-\mathrm{BO}$ to monitor treatment response, A2780 xenograft-bearing mice were treated with therapeutic doses of olaparib and imaged with ${ }^{18} \mathrm{~F}-\mathrm{BO}$ and ${ }^{18} \mathrm{~F}-\mathrm{FDG}$ before and after treatment. ${ }^{18} \mathrm{~F}-\mathrm{BO}$ showed a drop in tumor-to-muscle ratio from 3.8 to 1.3 after treatment with olaparib, whereas no changes were observed for ${ }^{18} \mathrm{~F}-\mathrm{FDG}$. 


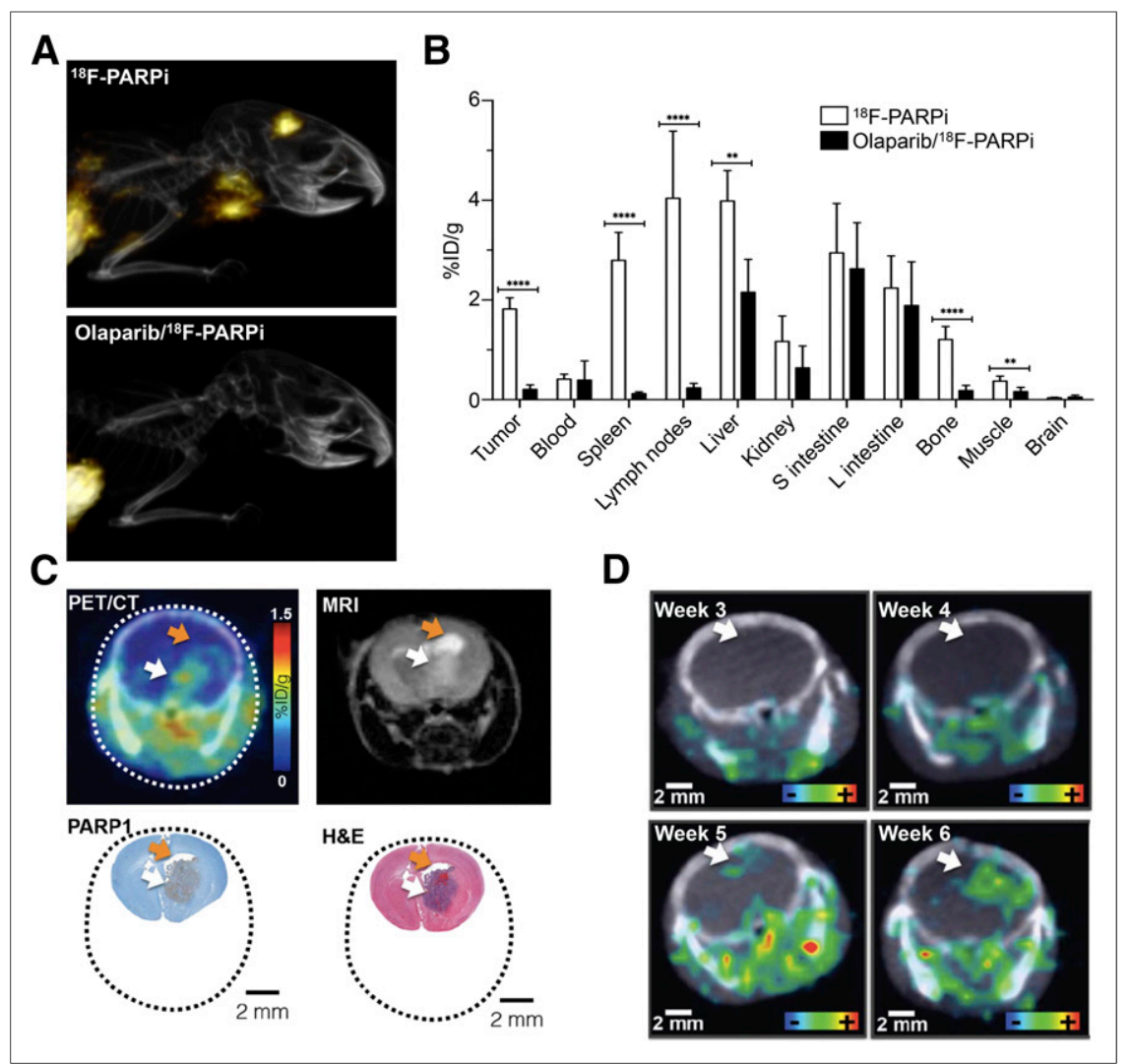

FIGURE 3. ${ }^{18} \mathrm{~F}-\mathrm{PARPi}$ in brain tumor models $(6,12)$. (A) Maximum-intensity projections from orthotopic U251 tumor-bearing mice. (B) ${ }^{18} \mathrm{~F}-\mathrm{PARPi}$ biodistribution $2 \mathrm{~h}$ after intravenous injection in U251 tumor-bearing athymic nude mice (subcutaneous xenograft). (C) ${ }^{18} \mathrm{~F}$-PARPi uptake in genetically engineered diffuse intrinsic pontine glioma mouse model, compared with MRI and histology. (D) Imaging of tumor growth progression in genetically engineered diffuse intrinsic pontine glioma mouse model. White arrows indicate tumor, and orange arrows accumulation of cerebrospinal fluid. H\&E = hematoxylin and eosin.

${ }^{18}$ F-PARPi-FL $(28,29)$ is a radiolabeled analog of PARPiFL and currently the only dual-modality PET/fluorescent PARP probe available. The radiosynthesis was achieved via Lewis acid-assisted fluoride exchange with either triflic anhydride (28) or tin tetrachloride (29). With this kind of reaction, it was only possible to produce ${ }^{18} \mathrm{~F}-\mathrm{PARPi}-\mathrm{FL}$ with a maximum specific activity of just $333 \mathrm{MBq} / \mu$ mol. Regardless, the PET images showed tumor uptake, and quantification of ${ }^{18} \mathrm{~F}-\mathrm{PARPi}-\mathrm{FL}$ in U87 glioblastoma xenografts at $2 \mathrm{~h}$ after injection showed a tumor SUV of 1.1. Blocking with a $50 \mathrm{mg} / \mathrm{kg}$ dose of olaparib reduced the SUV by $91 \%$, to 0.1 , which was a strong improvement compared with ${ }^{18} \mathrm{~F}-\mathrm{BO}$, for which blocking reduced the SUV by only $56 \%$. Dual-modality functionality was confirmed using epifluorescence imaging and autoradiography, for which similar tumor-to-brain ratios between the radioactive $(9: 1)$ and fluorescent (7:1) signals were found. However, PET and biodistribution data also revealed a relatively high signal in the bone $(>10$ percentage injected dose $[\% \mathrm{ID}] / \mathrm{g}$ ), indicating that ${ }^{18} \mathrm{~F}$-PARPi-FL suffered from defluorination in vivo.

${ }^{18} \mathrm{~F}-\mathrm{PARPi}(6,12)$ sacrificed BODIPY-FL for a highly stable fluorobenzene ring without negatively affecting up- take and specificity. ${ }^{18}$ F-PARPi uptake in orthotopic U251 glioblastoma xenografts (6) was increased to $2.2 \%$ ID/g $\quad\left({ }^{18} \mathrm{~F}-P A R P i-F L, \quad 0.8 \%\right.$ ID/g), with similarly high specificity $(87 \%$ blocking; ${ }^{18}$ F-PARPi-FL, $81 \%$ ) (Fig. 3A). Autoradiography revealed high tumorto-brain and tumor-to-muscle ratios (700:1 and 30:1, respectively). Biodistribution studies with subcutaneous xenografts (Fig. 3B) confirmed the uptake and specificity findings. Bone uptake was much lower than with ${ }^{18} \mathrm{~F}$-PARPiFL and could also be blocked by olaparib (83\% reduction in uptake), indicating specific uptake rather than defluorination. ${ }^{18} \mathrm{~F}-\mathrm{PARPi}$ was also evaluated for imaging of diffuse intrinsic pontine glioma in a genetically engineered mouse model (12). This malignancy is difficult to monitor with established methods and has seen limited progress in terms of treatment options over the past few decades (30). Autoradiography and PET imaging showed localized, specific uptake (Fig. 3C). ${ }^{18} \mathrm{~F}$-PARPi could also be used to monitor tumor progression noninvasively over several weeks (Fig. 3C), suggesting potential therapy-monitoring applications.

In addition to ${ }^{18} \mathrm{~F}$-PARPi, the iodinated compound ${ }^{124 / 131}$ I-PARPi $(14,31)$ $\left(\mathrm{IC}_{50}, 9 \mathrm{nM}\right)$ was developed. It is chemically identical to ${ }^{18} \mathrm{~F}$-PARPi except that

${ }^{131} \mathrm{I}$ (SPECT) or ${ }^{124} \mathrm{I}$ (PET) takes the place of ${ }^{18} \mathrm{~F}$ in the halogenated benzene ring. Like ${ }^{18} \mathrm{~F}-\mathrm{PARPi},{ }^{124 / 131}$ I-PARPi was first studied in orthotopic U251 tumors (14). Autoradiography showed high tumor-to-brain (16:1) and tumor-to-muscle (6:1) ratios and high specificity (65\% blocking). SPECT imaging with ${ }^{131}$ I-PARPi was possible, and PET quantification with ${ }^{124} \mathrm{I}$-PARPi showed $0.43 \% \mathrm{ID} / \mathrm{g}$ tumor uptake and a tumorto-brain ratio of 40:1. Another study (31) showed an alternative synthetic route to this radioiodinated PARP inhibitor with ${ }^{123} \mathrm{I}$ and ${ }^{125} \mathrm{I}$. The study presented a single-step iodination using a solid-state halogen exchange with a bromine leaving group, producing higher overall yields and specific activities.

\section{RADIOLABELED PROBES STRUCTURALLY SIMILAR TO RUCAPARIB}

Rucaparib $\left(\mathrm{K}_{\mathrm{i}}, 1.4 \mathrm{nM}(32)\right)$, the second Food and Drug Administration-approved PARP inhibitor, has also inspired radiolabeled analogs based on the former lead compound, AG14361 $\left(\mathrm{K}_{\mathrm{i}}, 5.8 \mathrm{nM}\right)$. AG14361-which has been shown to be specific to PARP1 (32) - and its derived radiotracers feature a benzimidazole instead of rucaparib's fluorinated 


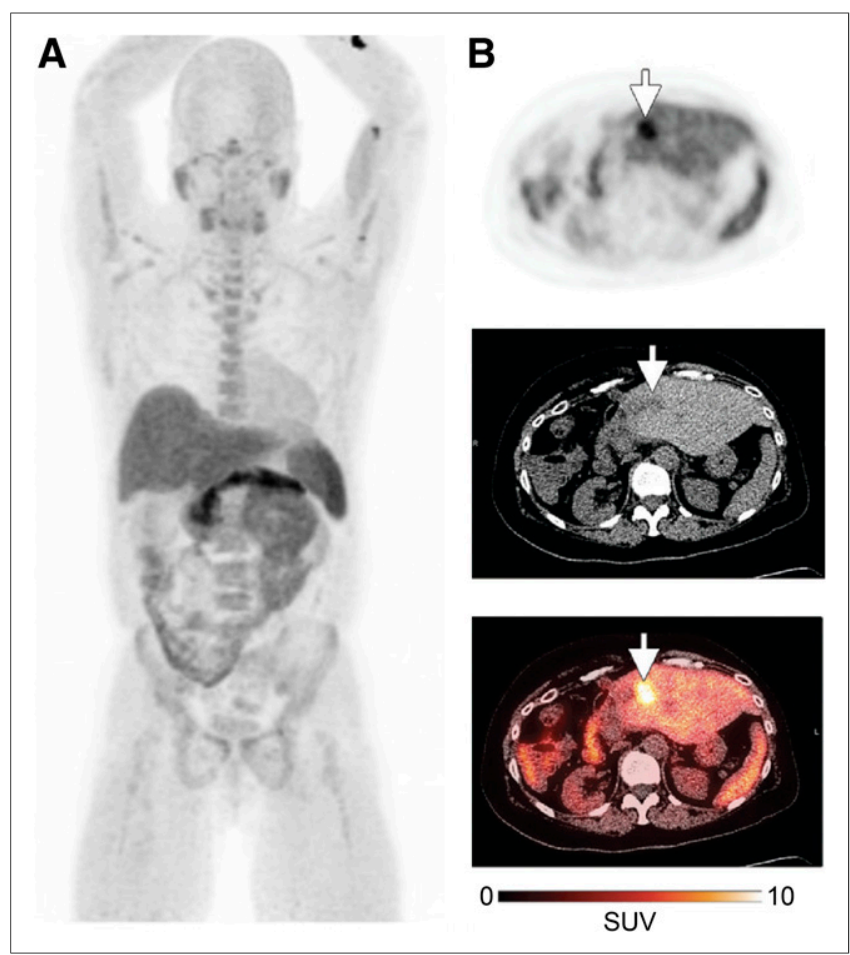

FIGURE 4. First-in-human ${ }^{18} \mathrm{~F}-\mathrm{FTT}$ study (35). (A) Representative maximum-intensity projection from healthy volunteer. (B) ${ }^{18} \mathrm{~F}-$ FTT uptake in liver metastasis (arrows) from patient with biphenotypic hepatocellular carcinoma and cholangiocarcinoma.

indole. ${ }^{18}$ F-FluorThanatrace $\left({ }^{18} \mathrm{~F}-\mathrm{FTT}\right)(23,33-35)\left(\mathrm{IC}_{50}, 6.3\right.$ $\mathrm{nM})$, which replaces dimethyl phenylmethanamine on AG14361 with ${ }^{18} \mathrm{~F}$-fluoroethoxy benzene, was first tested in MDA-MB-436 and MDA-MB-231 xenografts. PET imaging showed 3-5\%ID/g tumor uptake after $60 \mathrm{~min}$, which was reduced to $2 \% \mathrm{ID} / \mathrm{g}$ after olaparib blocking. ${ }^{18} \mathrm{~F}-\mathrm{FTT}$ uptake was imaged in a BRCA mutant tumor model (HCC1937) and compared with BRCA wild-type tumor models (MDA-MB231 and MCF7). In vitro ${ }^{18} \mathrm{~F}-\mathrm{FTT}$ uptake, reported as specific binding ratios in which nonspecific binding was subtracted, correlated with PARP1 and poly(adenosine diphosphateribose) levels, which were higher in BRCA mutated/mutant cells. PET imaging showed higher tumor-to-muscle ratios for HCC1937 (1.9:1) than for MDA-MB-231 (1.5:1) or MCF7 (1.2:1). A different study (34) compared in vitro ${ }^{18} \mathrm{~F}-\mathrm{FTT}$ uptake in BRCA mutant (SNU-251) and wild-type ovarian cancer cells (SKOV-3). It was shown that BRCA mutant SNU-251 cells were more sensitive than SKOV-3 cells to PARP inhibition and radiation therapy. These findings suggest that ${ }^{18} \mathrm{~F}-\mathrm{FTT}$ might be used to predict treatment efficacy of PARP inhibitor therapy, radiation, or a combination of the two.

In addition to ${ }^{18} \mathrm{~F}$-FTT, there are two iodinated $(36,37)$ compounds based on the benzimidazole scaffold of AG14361. ${ }^{125} \mathrm{I}-\mathrm{KX} 1\left(\mathrm{~K}_{\mathrm{i}},<8 \mathrm{nM}\right)$ is structurally identical to ${ }^{18}$ F-FTT except that ${ }^{125}$ I replaces the fluoroethoxy moiety $(36,37)$. It was shown in vitro that ${ }^{125} \mathrm{I}-\mathrm{KX} 1$ correlated with
PARP1 expression in a panel of breast and ovarian cancer cell lines but not with poly(adenosine diphosphate-ribose), as observed with ${ }^{18}$ F-FTT. Biodistribution showed that HCC1937 tumors had significantly higher uptake than MDA-MB-231 tumors, corresponding to PARP1 expression levels. Blocking with olaparib reduced ${ }^{125}$ I-KX1 uptake in HCC1937 tumors, but the difference was not statistically significant. No blocking was seen for MDA-MB-231. Autoradiographically, unblocked-to-blocked ratios of 2.7:1 for HCC1937 tumors and 0.92:1 for MDA-MB-231 tumors were found.

Another study (37) presented ${ }^{125} \mathrm{I}-\mathrm{KX}-02-019\left(\mathrm{~K}_{\mathrm{i}}, 13.9 \mathrm{nM}\right)$, a modified version of ${ }^{125} \mathrm{I}-\mathrm{KX} 1$ that features a bicyclic instead of a tricyclic benzimidazole. ${ }^{125} \mathrm{I}-\mathrm{KX}-02-019$ is a close analog of AG14032 (38) $\left(\mathrm{K}_{\mathrm{i}}, 6.7 \mathrm{nM}\right)$, another rucaparib predecessor, with its chloride replaced by ${ }^{125} \mathrm{I}$. In vitro studies showed that the specific binding ratios to PARP1 knockout mouse embryonic fibroblasts were higher for ${ }^{125} \mathrm{I}-\mathrm{KX}-02-019$ than for either ${ }^{18} \mathrm{~F}-\mathrm{FTT}$ or ${ }^{125} \mathrm{I}-\mathrm{KX} 1$. Biodistribution with EMT6 murine mammary carcinoma-bearing mice showed uptake close to $1 \% \mathrm{ID} / \mathrm{g}$ at $2 \mathrm{~h}$, with a tumor-to-muscle ratio of about 5 .

\section{PARP IMAGING IN THE CLINIC}

${ }^{18} \mathrm{~F}-\mathrm{FTT}$ is the first PARP-targeted radiotracer that has been tested in humans (NCT02469129) (35). A study on 8 healthy volunteers and 8 patients with a variety of malignancies found that a dose of $370 \mathrm{MBq}$ resulted in a total effective dose of $5.1 \mathrm{mSv}$ for men and $6.9 \mathrm{mSv}$ for women, with the highest uptake occurring in the spleen, pancreas, and liver. PET images showed increased uptake in tumor regions for two patients, one of whom had recurrent pancreatic ductal adenocarcinoma and the other biphenotypic hepatocellular carcinoma and cholangiocarcinoma (Fig. 4). In addition to this study, one phase I/II study recently opened to evaluate PARPi-FL for early detection of oral cancer after topical application (NCT03085147). The addition of molecularly targeted fluorescence contrast for visual tumor detection and navigation during surgery may represent an asset in enabling physicians to discriminate healthy tissue from malignant growth.

\section{CONCLUSION}

PARP has been shown to be an interesting and valuable target for imaging. A wide variety of cancer types has been imaged, with diverse clinical applications in mind. These include fluorescence-assisted screening and diagnosis, intraoperative fluorescence-guided surgery, and PET-guided patient stratification and treatment monitoring. The first PARP imaging agent was reported a mere 12 years ago, and two compounds are now in clinical trials. Although, preclinically, PARP imaging agents could become powerful tools for understanding the biology behind the DNA damage response system, the ultimate translational and clinical aims of these agents are to provide a real and tangible improvement in overall patient care. 


\section{DISCLOSURE}

This work was supported by National Institutes of Health grants NIH R01CA204441 (to Thomas Reiner), R21CA191679 (to Thomas Reiner), and P30 CA008748. We thank the Center for Molecular Imaging and Nanotechnology, the Tow Fellowship Program in Molecular Imaging and Nanotechnology (for support to Brandon Carney and Susanne Kossatz), the National Science Foundation (Integrative Graduate Education and Research Traineeship 0965983 for Brandon Carney), and the German Research Foundation (for support to Susanne Kossatz).

\section{REFERENCES}

1. Scott CL, Swisher EM, Kaufmann SH. Poly (ADP-ribose) polymerase inhibitors: recent advances and future development. J Clin Oncol. 2015;33:1397-1406.

2. Michels J, Vitale I, Saparbaev M, Castedo M, Kroemer G. Predictive biomarkers for cancer therapy with PARP inhibitors. Oncogene. 2014;33:3894-3907.

3. Pommier Y, O'Connor MJ, de Bono J. Laying a trap to kill cancer cells: PARP inhibitors and their mechanisms of action. Sci Transl Med. 2016;8:362ps17.

4. Knight JC, Koustoulidou S, Cornelissen B. Imaging the DNA damage response with PET and SPECT. Eur J Nucl Med Mol Imaging. 2017;44:1065-1078.

5. Agasti SS, Laughney AM, Kohler RH, Weissleder R. A photoactivatable drugcaged fluorophore conjugate allows direct quantification of intracellular drug transport. Chem Commun (Camb). 2013;49:11050-11052.

6. Carney B, Carlucci G, Salinas B, et al. Non-invasive PET imaging of PARP1 expression in glioblastoma models. Mol Imaging Biol. 2016;18:386-392.

7. Dubach JM, Vinegoni C, Mazitschek R, Fumene Feruglio P, Cameron LA, Weissleder R. In vivo imaging of specific drug-target binding at subcellular resolution. Nat Commun. 2014;5:3946.

8. Dubach JM, Kim E, Yang K, et al. Quantitating drug-target engagement in single cells in vitro and in vivo. Nat Chem Biol. 2017;13:168-173.

9. Irwin CP, Portorreal Y, Brand C, et al. PARPi-FL: a fluorescent PARP1 inhibitor for glioblastoma imaging. Neoplasia. 2014;16:432-440.

10. Kossatz S, Weber WA, Reiner T. Optical imaging of PARP1 in response to radiation in oral squamous cell carcinoma. PLoS One. 2016;11:e0147752.

11. Kossatz S, Brand C, Gutiontov S, et al. Detection and delineation of oral cancer with a PARP1 targeted optical imaging agent. Sci Rep. 2016;6:21371.

12. Kossatz S, Carney B, Schweitzer M, et al. Biomarker-based PET imaging of diffuse intrinsic pontine glioma in mouse models. Cancer Res. 2017;77:2112-2123.

13. Reiner T, Lacy J, Keliher EJ, et al. Imaging therapeutic PARP inhibition in vivo through bioorthogonally developed companion imaging agents. Neoplasia. 2012; 14:169-177.

14. Salinas B, Irwin CP, Kossatz $\mathrm{S}$, et al. Radioiodinated PARP1 tracers for glioblastoma imaging. EJNMMI Res. 2015;5:123.

15. Thurber GM, Yang KS, Reiner T, et al. Single-cell and subcellular pharmacokinetic imaging allows insight into drug action in vivo. Nat Commun. 2013;4:1504.

16. Thurber GM, Reiner T, Yang KS, Kohler RH, Weissleder R. Effect of smallmolecule modification on single-cell pharmacokinetics of PARP inhibitors. Mol Cancer Ther. 2014;13:986-995.

17. Deeks ED. Olaparib: first global approval. Drugs. 2015;75:231-240.

18. Menear KA, Adcock C, Boulter R, et al. 4-[3-(4-cyclopropanecarbonylpiperazine1-carbonyl)-4-fluorobenzyl]-2H-phthalazin-1-one: a novel bioavailable inhibitor of poly(ADP-ribose) polymerase-1. J Med Chem. 2008;51:6581-6591.
19. Reiner T, Earley S, Turetsky A, Weissleder R. Bioorthogonal small-molecule ligands for PARP1 imaging in living cells. Chembiochem. 2010;11:23742377.

20. Rutkowska A, Thomson DW, Vappiani J, et al. A modular probe strategy for drug localization, target identification and target occupancy measurement on single cell level. ACS Chem Biol. 2016;11:2541-2550.

21. Kim E, Yang KS, Giedt RJ, Weissleder R. Red Si-rhodamine drug conjugates enable imaging in GFP cells. Chem Commun (Camb). 2014;50:4504-4507.

22. Tu Z, Chu W, Zhang J, Dence CS, Welch MJ, Mach RH. Synthesis and in vivo evaluation of $\left[{ }^{11} \mathrm{C}\right] \mathrm{PJ} 34$, a potential radiotracer for imaging the role of PARP-1 in necrosis. Nucl Med Biol. 2005;32:437-443.

23. Zhou $\mathrm{D}, \mathrm{Chu} \mathrm{W}, \mathrm{Xu}$ J, et al. Synthesis, $\left[{ }^{18} \mathrm{~F}\right]$ radiolabeling, and evaluation of poly (ADP-ribose) polymerase-1 (PARP-1) inhibitors for in vivo imaging of PARP-1 using positron emission tomography. Bioorg Med Chem. 2014;22:1700-1707.

24. Garcia Soriano F, Virág L, Jagtap P, et al. Diabetic endothelial dysfunction: the role of poly(ADP-ribose) polymerase activation. Nat Med. 2001;7:108-113.

25. Riss PJ, Soskic V, Schrattenholz A, Roesch F. Synthesis and radiosynthesis of N5- $\left[{ }^{18} \mathrm{~F}\right]$ fluoroethyl-pirenzepine and its metabolite N5-[ $\left.{ }^{18} \mathrm{~F}\right]$ fluoroethyl-LS 75. J Labelled Comp Radiopharm. 2009;52:576-579.

26. Keliher EJ, Reiner T, Turetsky A, Hilderbrand SA, Weissleder R. High-yielding, two-step ${ }^{18} \mathrm{~F}$ labeling strategy for ${ }^{18} \mathrm{~F}-\mathrm{PARP} 1$ inhibitors. ChemMedChem. 2011;6: $424-427$.

27. Reiner T, Keliher EJ, Earley S, Marinelli B, Weissleder R. Synthesis and in vivo imaging of a ${ }^{18} \mathrm{~F}$-labeled PARP1 inhibitor using a chemically orthogonal scavenger-assisted high-performance method. Angew Chem Int Ed Engl. 2011;50; 1922-1925.

28. Keliher EJ, Klubnick JA, Reiner T, Mazitschek R, Weissleder R. Efficient acidcatalyzed ${ }^{18} \mathrm{~F} /{ }^{19} \mathrm{~F}$ fluoride exchange of BODIPY dyes. ChemMedChem. 2014;9:1368-1373.

29. Carlucci G, Carney B, Brand C, et al. Dual-modality optical/PET imaging of PARP1 in glioblastoma. Mol Imaging Biol. 2015;17:848-855.

30. Warren KE. Diffuse intrinsic pontine glioma: poised for progress. Front Oncol. 2012;2:205.

31. Zmuda F, Malviya G, Blair A, et al. Synthesis and evaluation of a radioiodinated tracer with specificity for poly(ADP-ribose) polymerase-1 (PARP-1) in vivo. $J$ Med Chem. 2015;58:8683-8693.

32. Calabrese CR, Almassy R, Barton S, et al. Anticancer chemosensitization and radiosensitization by the novel poly(ADP-ribose) polymerase-1 inhibitor AG14361. J Natl Cancer Inst. 2004;96:56-67.

33. Edmonds CE, Makvandi M, Lieberman BP, et al. $\left[{ }^{18} \mathrm{~F}\right]$ FluorThanatrace uptake as a marker of PARP1 expression and activity in breast cancer. Am J Nucl Med Mol Imaging. 2016;6:94-101.

34. Sander Effron S, Makvandi M, Lin L, et al. PARP-1 expression quantified by $\left[{ }^{18} \mathrm{~F}\right]$ FluorThanatrace: a biomarker of response to PARP inhibition adjuvant to radiation therapy. Cancer Biother Radiopharm. 2017;32:9-15.

35. Michel LS, Dyroff S, Brooks FJ, et al. PET of poly (ADP-ribose) polymerase activity in cancer: preclinical assessment and first in-human studies. Radiology. 2017;282:453-463.

36. Makvandi M, Xu K, Lieberman BP, et al. A radiotracer strategy to quantify PARP-1 expression in vivo provides a biomarker that can enable patient selection for PARP inhibitor therapy. Cancer Res. 2016;76:4516-4524.

37. Anderson RC, Makvandi M, Xu K, et al. Iodinated benzimidazole PARP radiotracer for evaluating PARP1/2 expression in vitro and in vivo. Nucl Med Biol. 2016;43:752-758.

38. Thomas HD, Calabrese CR, Batey MA, et al. Preclinical selection of a novel poly(ADP-ribose) polymerase inhibitor for clinical trial. Mol Cancer Ther. 2007;6:945-956. 